\title{
Simulation Based Design Analysis of an Adjustable Window Function
}

\author{
Orvila Sarker $^{1 *}$, Rezaul Huque Khan ${ }^{2}$ \\ ${ }^{1}$ Department of Information \& Communication Technology, Comilla University, Comilla, Bangladesh \\ ${ }^{2}$ Department of Applied Physics, Electronics \& Communication Engineering, University of Chittagong, Chittagong, Bangladesh \\ Email: ${ }^{*}$ orvilasarker@cou.ac.bd
}

How to cite this paper: Sarker, O. and Khan, R.H. (2016) Simulation Based Design Analysis of an Adjustable Window Function. Journal of Signal and Information Processing, 7, 214-226. http://dx.doi.org/10.4236/jsip.2016.74019

Received: August 5, 2016

Accepted: November 1, 2016

Published: November 4, 2016

Copyright $\odot 2016$ by authors and Scientific Research Publishing Inc. This work is licensed under the Creative Commons Attribution International License (CC BY 4.0).

http://creativecommons.org/licenses/by/4.0/

\section{(c) (7) Open Access}

\begin{abstract}
Window based Finite Impulse Response filters have the problem that in order to obtain better performance from these filters in terms of minimum stopband attenuation cost has to be paid for half main-lobe width and vice-versa. A solution of this contradictory behavior is to increase the length of the window which in turn requires more hardware hence increasing the cost of system. This paper proposes a novel window based on two shifted hyperbolic tangent functions. The proposed window contains an adjustable parameter, with the help of which desired time and frequency domain characteristics may be achieved for relatively shorter window length. The characteristics of the proposed window are compared with those of the two well-known adjustable windows namely Cosh window and Exponential window. MATLAB simulation results show that for the same value of window length, the proposed window provides improved output, and thus it makes a good compromise between minimum stopband attenuation and half main-lobe width compared to the windows mentioned previously.
\end{abstract}

\section{Keywords}

Finite Impulse Response Filter, Minimum Stopband Attenuation, Half Main-Lobe Width

\section{Introduction}

Ideal filters have perfectly flat passband with infinite attenuation in the stopband. Unfortunately these filters are practically unrealizable because of their infinite number of co-efficients in the time domain. Hence in order to implement them, the co-efficients must be truncated [1]. Figure 1 shows the truncation of ideal impulse response $h_{d}(n)$ by a finite length sequence $W(n)$ whose values range from 0 to $(N-1)$ to get a Finite Im- 
pulse Response $h(n)$ having a length of $N$. However direct truncation results in unwanted ripples in the passband commonly known as "Gibbs phenomenon" as shown in Figure 2 .

This is because multiplication in the time domain (Figure 2(a)) is equivalent to circular convolution in the frequency domain (Figure 2(b)). Moreover, this is the main reason for the contradiction between minimum stopband attenuation and main lobe width of windowed filters. From Figure 2(b) it is clear that there are unwanted ripples in the resultant frequency response curve of the window. Also, the transition width becomes wider compared to the ideal case. Rather than direct truncation, a smoothly taper curve known as "Window" is used [1]. It is the shape of the window that necessarily determines whether it results in better stopband attenuation or better transition width or both of the designed filters.

Windows may be fixed or adjustable. Fixed windows have only one parameter i.e. window length. In order to get better performance from fixed windows, the only way is to increase the window length which in terns increases the cost of the resultant system. On the other hand adjustable windows have two or more adjustable parameters that provide extra degree of freedom to control time and frequency domain characteristics [2].

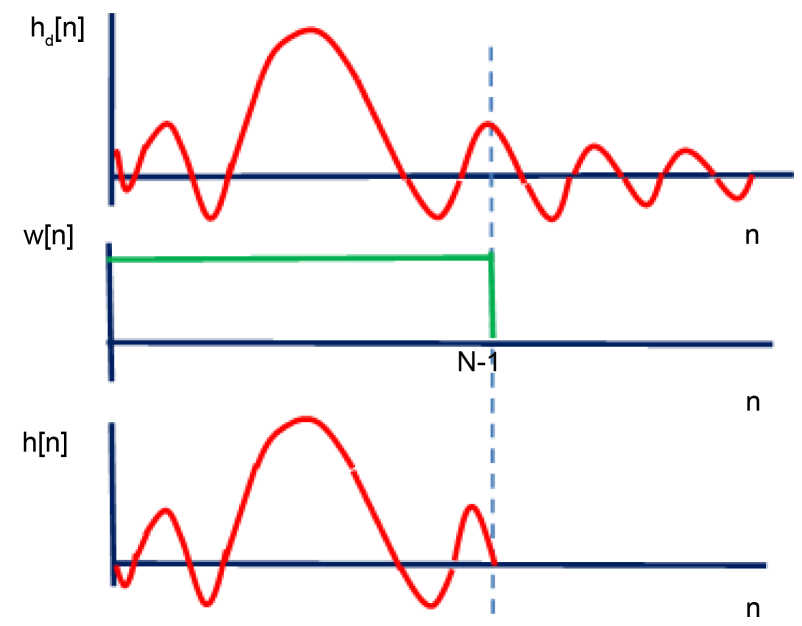

Figure 1. Windowing process.

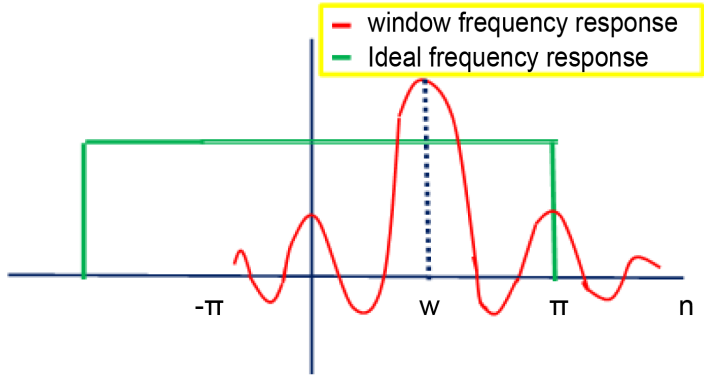

(a)

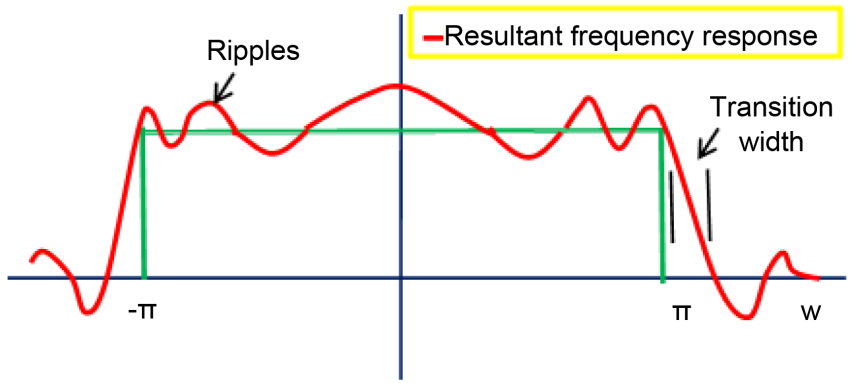

(b)

Figure 2. Effect of direct truncation-Gibb's phenomenon. 
Several windows whether fixed or adjustable, have been introduced in the field of digital signal processing [2]-[10]. Based on Blackman window function an efficient adjustable window has been proposed in [3]. This window provides better minimum stop band attenuation compared to Hamming and Hanning window for some appropriate values of adjustable parameter; however Hamming window remains superior in terms of main lobe width [3]. Using modified coefficient of Hamming window, a fixed window function having lower main lobe width compared to Hamming window has been proposed in [4]. A three-parameter Ultraspherical window has been proposed in [5]. For some particular values of window length, Ultraspherical window shows better result in terms of ripple ratio compared to Kaiser window, otherwise Kaiser window remains better [5]. A fixed window function has been proposed in [5]. Although this window produces better minimum stopband attenuation compared to Hamming and Kaiser windows but it incorporates with worse half main lobe width [6]. An adjustable exponential window has been proposed in [7]. For same window length and main lobe width, this exponential window results in smaller stopband attenuation compared to Kaiser window and better stopband attenuation compared to Ultraspherical window [7]. An adjustable window based on Cosine hyperbolic function derived from the Kaiser window has been proposed in [8]. For an efficient value of adjustable parameter, this window function provides greater stopband attenuation compared to Kaiser window; however the main lobe half width remains the same [8]. Thus we find that windowed filters have the characteristic that they show contradictory nature between minimum stopband attenuation and main lobe width. This contradictory behavior makes them inefficient in many of the signal processing applications where both minimum stopband attenuation and main lobe width cannot be compromised.

Stopband attenuation is an important spectral parameter. For filter design applications, it is required to have good stopband attenuation in order to adequately block the unwanted frequencies. Better minimum stopband attenuation increases the ability of detecting weak signals in the presence of strong narrowband signals. Main-lobe width is another important parameter for some applications, such as frequency spectral analysis of signal. A narrower main-lobe width means that it distinguishes two closely spaced frequencies more accurately [1].

As mentioned previously, it has been proposed that for the same window length and main-lobe width, the exponential window provide better side roll-off ratio but worse ripple ratio and Cosh window provides better minimum stopband attenuation compared to Kaiser window [7] [8]. For zero value of adjustable parameter, both Cosh and Exponential windows behave like rectangular window providing narrower main-lobe half width. It is desirable for some applications to obtain greater minimum stopband attenuation compared to these two windows while having the same main-lobe half width [7] [8].

A novel window based on hyperbolic tangent function is proposed in this article. For some values of adjustable parameter, this proposed window provides increased stopband attenuation while the half main lobe width remains unchanged or even gets better 
compared to some well known windows. In order to show the efficiency of our proposed window, we compare the performance of proposed window with that of Cosh window and Exponential window. Simulation results show that for smaller values of adjustable parameter of both Cosh and Exponential windows, the proposed window provides greater minimum stopband attenuation for the same window length and equal or narrower main-lobe half width. Hence, our proposed window makes a good compromise between stopband attenuation and main lobe width. Design relationship among adjustable parameter, window length and stopband attenuation are shown and two important design equations are provided. These design equations would be helpful in order to select the appropriate value of adjustable parameter of our proposed window for desired window length and stopband attenuation. The filtering action of the proposed window is also analyzed using MATLAB simulator. Significant suppression of unwanted high frequency components in the frequency domain by our proposed window has been observed.

\section{Derivation of Proposed Window}

Two shifted hyperbolic functions as hyperbolic window has been used for the development of frequency selective filters in the frequency domain. Using the scaling and shifting properties of Fourier Transform, it has been showed that within a certain limit, these two shifted hyperbolic tangent function approximates a Sinc function in the time domain, hence close to ideal filter [9] [10].

In this paper to derive our proposed window we combine two hyperbolic tangent functions in the time domain rather than the frequency domain. The proposed window is defined as

$$
w(n)=5 \beta\left[\tanh \frac{\left(\left(n-\frac{N-1}{2}\right)+0.1\right)}{\beta}-\tanh \frac{\left(\left(n-\frac{N-1}{2}\right)-0.1\right)}{\beta}\right]
$$

where $N$ is the length of window and $\beta$ is the adjustable parameter $(\beta \neq 0)$.

\section{Simulation Results and Performance Analysis}

Different performance characteristics and the corresponding data are obtained using MATLAB software. For simplicity we have considered small values of window length for analyzing the response of our proposed window. But the performance of our proposed window would be the same for larger window length.

\subsection{Proposed Window for Different Values of Adjustable Parameter}

Figure 3 shows the frequency response characteristics of proposed window for a fixed value of window length, $N=30$. From Figure 1 it is clearly observed that for a fixed $N$ lower value of $\beta$ results better minimum stopband attenuation $(A s)$ which is desirable. At the same time it is incorporated with wider main-lobe half width. On the other side a narrower main-lobe half width can be obtained for higher values of $\beta$ with poorer 
minimum stopband attenuation. Thus for a given window length, higher values of $\beta$ results good mainlobe width and lower values of $\beta$ results good minimum stopband attenuation which maintains the contradictory nature of windowed filters. The corresponding minimum stopband attenuation $(A s)$ and half main-lobe width $\left(W_{R}\right)$ for $\beta=$ 12, 16, 20 are tabulated in Table 1.

From Table 1 it is observed that when $\beta=12$, a minimum stopband attenuation of $-43.19 \mathrm{~dB}$ is obtained. If we increase $\beta$ to 16 , stopband attenuation becomes -28.40 $\mathrm{dB}$ and increasing $\beta$ from 16 to 20 results a stopband attenuation of $-26.18 \mathrm{~dB}$. Thereby we get better minimum stopband attenuation for lower values of $\beta$. But this lower $\beta$ results worse mainlobe width which is undesirable. Again, increasing $\beta$ from 12 to 20 , the normalized mainlobe width becomes narrower and narrower from 0.171 to 0.140 .

Table 2 shows some maximum values of stopband attenuation that results for a given window length. For example, when $N=40$, we are able to get a maximum value of $A s=-104.27 \mathrm{~dB}$ which is more than enough for such a small window length in many practical applications.

\subsection{Relationship between Adjustable Parameter and Minimum Stopband Attenuation}

In the frequency domain, stopband attenuation is one of the most important parameter

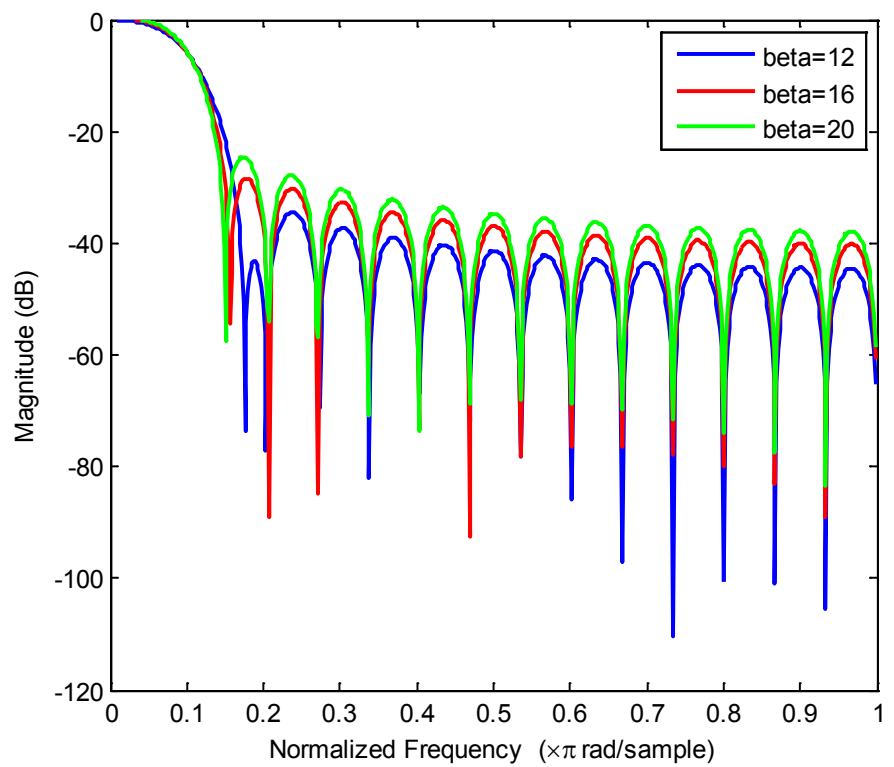

Figure 3. Frequency response characteristics of proposed window.

Table 1. Characteristic values of proposed window.

\begin{tabular}{cccc}
\hline$N$ & $\beta$ & $A s(\mathrm{~dB})$ & $W_{R}$ \\
\hline 30 & 12 & -43.19 & 0.171 \\
30 & 16 & -28.40 & 0.154 \\
30 & 20 & -26.18 & 0.140 \\
\hline
\end{tabular}


of a designed filter. Figure 4 shows the relationship between minimum stopband attenuation $(A s)$ and adjustable parameter $(\beta)$ for window length $N=30,40,50,60,70$, $80,90,100$.

For fixed values of $N$, a general approximate design relation between $\beta$ and $A s$ can be achieved using quadratic polynomial curve fitting method which is given by Equation (2). This equation will be very helpful for the designers who want to use this window for a desired value of $N$ and $A s$.

$$
\beta=a A s^{4}+b A s^{3}+c A s^{2}+d A s+e
$$

Values of co-efficients $a, b, c, d$, e in Equation (2) are given in Table 3 for $N=30,40$, $50,60,70,80,90,100$. We have taken the values of $A s=-20 \mathrm{~dB},-25 \mathrm{~dB},-30 \mathrm{~dB},-35$

Table 2. Maximum achievable values of $A s$ for some $N$.

\begin{tabular}{ll}
\hline$N$ & $A s($ in dB) \\
\hline 10 & -34.10 \\
20 & -75.07 \\
30 & -85.81 \\
40 & -104.27 \\
50 & -110.05 \\
60 & -144.64 \\
70 & -145.87 \\
80 & -155.44 \\
90 & -156.56 \\
100 & -182.58 \\
\hline
\end{tabular}

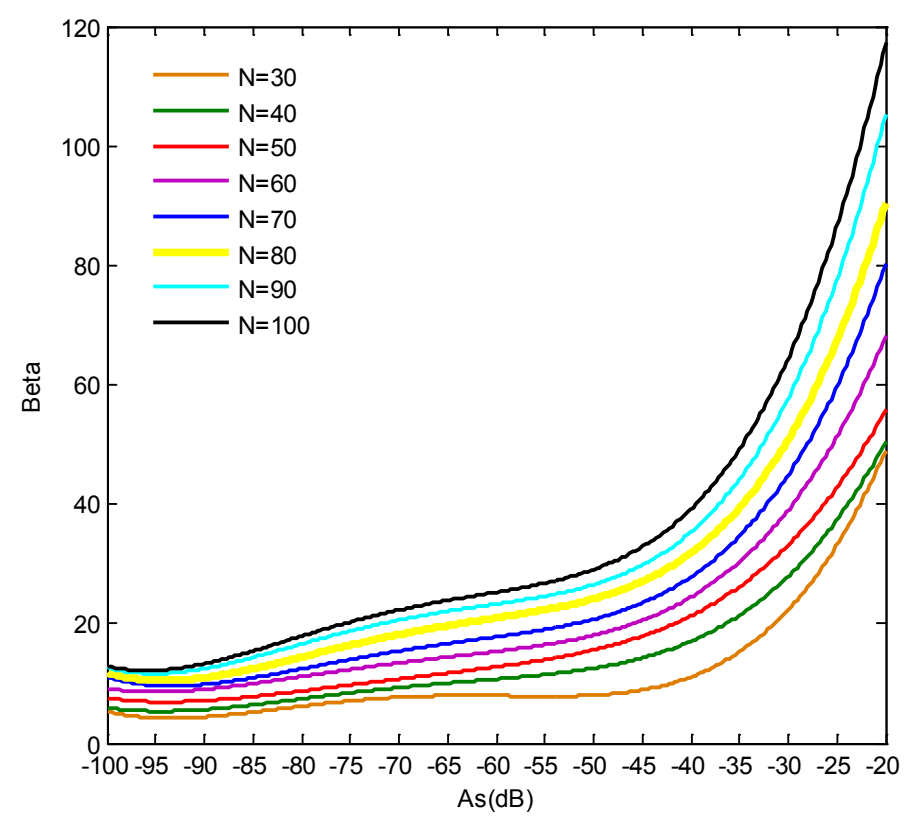

Figure 4. The relationship between minimum stopband attenuation $(A s)$ and adjustable parame$\operatorname{ter}(\beta)$. 
$\mathrm{dB},-40 \mathrm{~dB},-45 \mathrm{~dB},-50 \mathrm{~dB},-55 \mathrm{~dB},-60 \mathrm{~dB},-65 \mathrm{~dB},-70 \mathrm{~dB},-75 \mathrm{~dB},-80 \mathrm{~dB},-85$

$\mathrm{dB},-90 \mathrm{~dB},-95 \mathrm{~dB},-100 \mathrm{~dB}$ and obtained the corresponding values of $\beta$ using Equation (2). From Figure 4 it is clear that in order to obtain a stopband attenuation of -100 $\mathrm{dB}$ we require approximate values of $\beta=5,6,7,9,11,12,13,14$ for $N=30,40,50,60$, $70,80,90,100$ respectively. Again we observe that for $N=30$, a stopband attenuation of $-20 \mathrm{~dB}$ is obtained when $\beta=48$ and a stopband attenuation of $-100 \mathrm{~dB}$ is obtained when $\beta=14$.

\subsection{Relationship between Adjustable Parameter and Window Length}

In some applications window length $N$ is kept constant. To obtain an approximate value of $\beta$ for a given $N$ and $A s$, windows of length $N=20,30,40,50,60,70,80,90,100$ were designed to cover the range $-25 \mathrm{~dB} \leq \mathrm{As} \leq-80 \mathrm{~dB}$. Figure 5 shows the plots of

Table 3. Approximate value of co-efficients appeared in Equation (2).

\begin{tabular}{cccccc}
\hline$N$ & $a$ & $b$ & $c$ & $d$ & $e$ \\
\hline 30 & $8.85134 \mathrm{E}-06$ & 0.0023954 & 0.24565 & 10.945 & 187.36 \\
40 & $5.9548 \mathrm{E}-06$ & 0.0016918 & 0.17729 & 8.2839 & 157.78 \\
50 & $5.1347 \mathrm{E}-06$ & 0.0014723 & 0.15769 & 7.6756 & 157.13 \\
60 & $7.0379 \mathrm{E}-06$ & 0.0020263 & 0.21601 & 10.314 & 203.08 \\
70 & $9.612 \mathrm{E}-06$ & 0.0027062 & 0.2815 & 13.084 & 249.42 \\
80 & $1.122 \mathrm{E}-05$ & 0.003149 & 0.32538 & 14.984 & 283.43 \\
90 & $1.303 \mathrm{E}-05$ & 0.0037011 & 0.38619 & 17.867 & 335.64 \\
100 & $1.3884 \mathrm{E}-05$ & 0.00397048 & 0.41821 & 19.529 & 370.09 \\
\hline
\end{tabular}

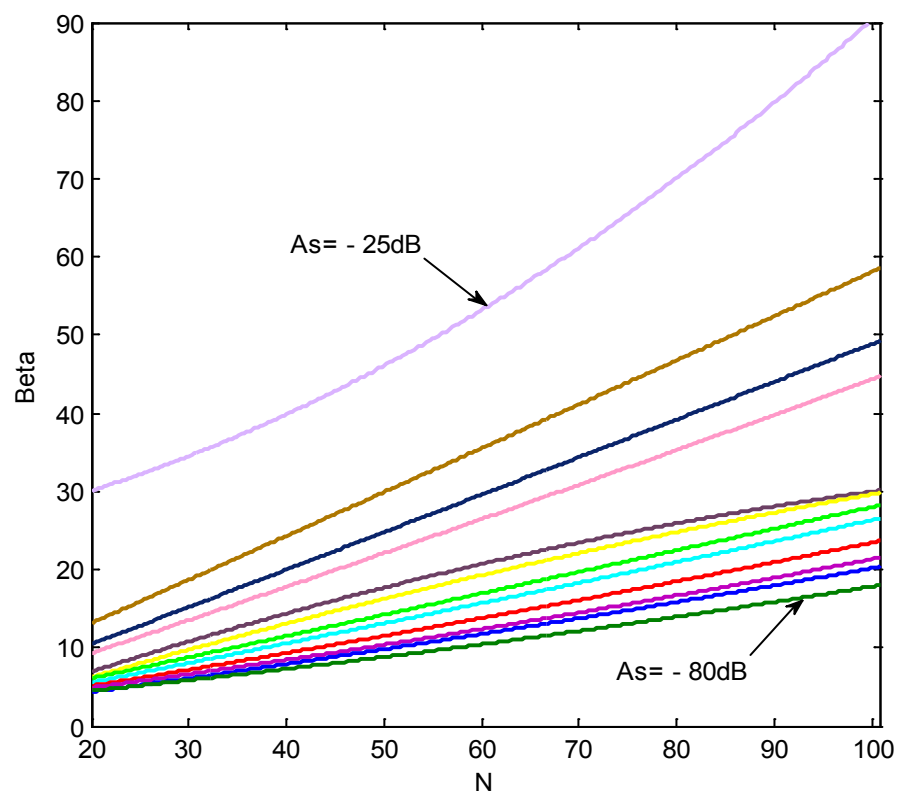

Figure 5. The relationship between window length, $N$ and adjustable parameter, $\beta$. 
window length $N$ and adjustable parameter $\beta$. Using quadratic polynomial curve fitting technique, an approximate design equation between $\beta$ and $N$ takes the general form,

$$
\beta=x N^{2}+y N+z
$$

Values of co-efficients $x, y, z$ in Equation (3) for a range of $A s$ are presented in Table 4.

Figure 5 shows that for a small value of $N$, very small changes in $\beta$ causes significant changes in stopband attenuation. For example when $N=20$, values of $\beta=10.5,9.2,7$, 6.9, 6.2, 6.1, 5.5, 5.1, 4.9, 4.3, 4.2, 4.1, 3.8, 3.2, 3 results stopband attenuation of $A s=-30$ $\mathrm{dB},-35 \mathrm{~dB},-40 \mathrm{~dB},-45 \mathrm{~dB},-50 \mathrm{~dB},-55 \mathrm{~dB},-60 \mathrm{~dB},-65 \mathrm{~dB},-70 \mathrm{~dB},-75 \mathrm{~dB},-80$ $\mathrm{dB},-85 \mathrm{~dB},-90 \mathrm{~dB},-95 \mathrm{~dB},-100 \mathrm{~dB}$ respectively. But from it is also clear that $A s=-25$ $\mathrm{dB}$ line is largely separated from other lines. This is because it is the lower limit of stopband attenuation that can be achieved by the proposed window. Again for some applications if we choose $N=20$ and want a value of $A s=-80 \mathrm{~dB}$, we need to select $\beta=$ 3 and if we choose $N=100$ and want a value of $A s=-80 \mathrm{~dB}$, we need to select $\beta=12$ which is easily visible from Figure 5.

\section{Performance Comparisons}

In order to show the efficiency of proposed window, it is compared with well-known Cosh window and Exponential window. Although adjustable parameters of these two windows posses different characteristic values compared to the adjustable parameter of our proposed window, for the sake of comparison, $\beta$ of the proposed window is chosen carefully that results a slightly better performance than the Cosh window and Exponential window.

\subsection{Comparison between Cosh Window and Proposed Window}

Kaiser window has been derived by modifying the zero order Bessel function of first

Table 4. Approximate value of co-efficients appeared in Equation (3) for some desired As.

\begin{tabular}{cccc}
\hline$A s($ in dB $)$ & $x$ & $y$ & $z$ \\
-80 & 0.00045 & 0.11 & 2.1 \\
-75 & 0.00031 & 0.16 & 0.99 \\
-70 & 0.00045 & 0.15 & 1.7 \\
-65 & 0.00031 & 0.19 & 1.2 \\
-60 & 0.00014 & 0.24 & 0.65 \\
-55 & $4.5 \mathrm{E}-05$ & 0.27 & 0.69 \\
-50 & -0.00084 & 0.39 & -1.3 \\
-45 & -0.001418 & 0.4585 & -1.681 \\
-40 & 0.00022 & 0.4109 & 1.0119 \\
-35 & $4.329 \mathrm{E}-05$ & 0.47481 & 0.938 \\
-30 & $5.411 \mathrm{E}-05$ & 0.5517 & 2.0413 \\
-25 & 0.0044 & 0.23 & 24 \\
\hline
\end{tabular}


kind. This modified Bessel function exhibits the same shape characteristics as the Cosh function. Thereby Cosh window has been defined as [8],

$$
w(n)=\frac{\operatorname{Cosh}(\alpha) \sqrt{1-\left(\frac{2 n}{N-1}\right)^{2}}}{\operatorname{Cosh}(\alpha)} \quad 0 \leq n \leq N-1
$$

Table 5 shows the corresponding data of the two windows for window length $N=$ 20. It is clear that, for different values of adjustable parameters $\alpha$ and $\beta$, the proposed window provides slightly greater minimum stopband attenuation than Cosh window for almost equal main-lobe half width. For example, when $\alpha=0$ and $\beta=50$ our proposed window results better minimum stopband attenuation and equal mainlobe half width compared to Cosh window. The same performance is obtained for $\alpha=1$ and $\beta=$ 14. But for $\alpha=2$ and $\beta=8$ our proposed window remains superior in terms of stopband attenuation only. Thus for higher values of $\beta$ better performance of our proposed window is more significant.

It is clearly observed from the Figures 6(a)-(c) that, for all pairs of $\alpha$ and $\beta$, our proposed window results better minimum stopband attenuation and half mainlobe width simultaneously which is very rare for most of the existing window filters. For small system (i.e., small window length $N$ ) the difference between characteristic values obtained by Cosh and proposed window may not be significant enough but for larger values of $N$ it would be more advantageous to use our proposed window other than Cosh window.

\subsection{Comparison between Exponential Window and Proposed Window}

The shape characteristics of zero order Bessel function is also similar to that of exponential function. Hence Exponential window has been defined as [7],

$$
w(n)=\frac{\exp (\alpha) \sqrt{1-\left(\frac{2 n}{N-1}\right)^{2}}}{\exp (\alpha)}|n| \leq \frac{N-1}{2}
$$

Figure 7 shows the frequency response characteristic comparison curve between Exponential window and proposed window. For all values of adjustable parameter, proposed window make a good compromise between the contradictory parameters

Table 5. Comparison data for Cosh window and proposed window.

\begin{tabular}{cccc}
\hline Window & $N$ & $A s$ (in dB) & $W_{R}$ \\
\hline Cosh $(\alpha=0)$ & 20 & -19.59 & 0.20 \\
Proposed $(\beta=50)$ & 20 & -20.58 & 0.20 \\
Cosh $(\alpha=1)$ & 20 & -25.32 & 0.22 \\
Proposed $(\beta=14)$ & 20 & -26.05 & 0.22 \\
Cosh $(\alpha=2)$ & 20 & -36.00 & 0.25 \\
Proposed $(\beta=8)$ & 20 & -36.26 & 0.26 \\
\hline
\end{tabular}




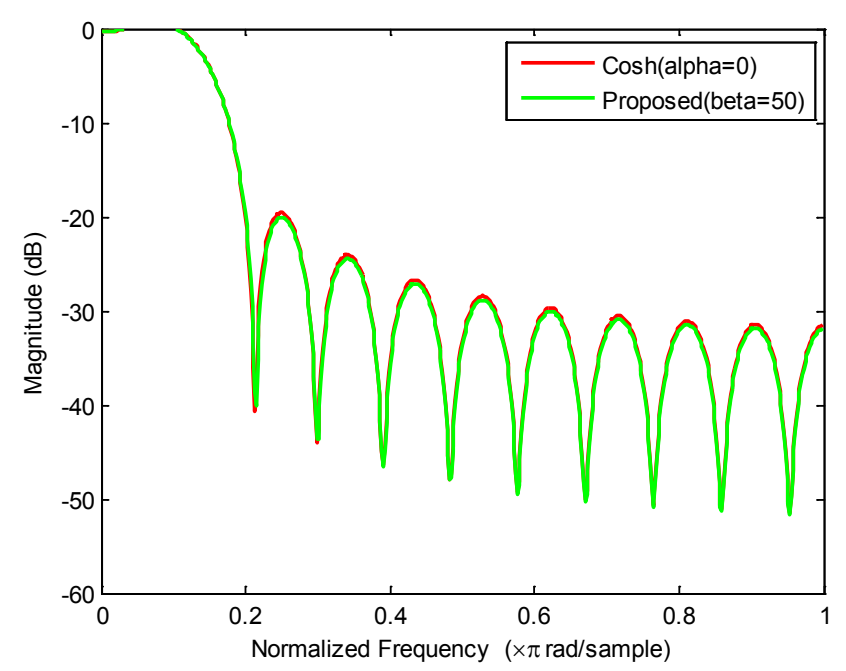

(a)

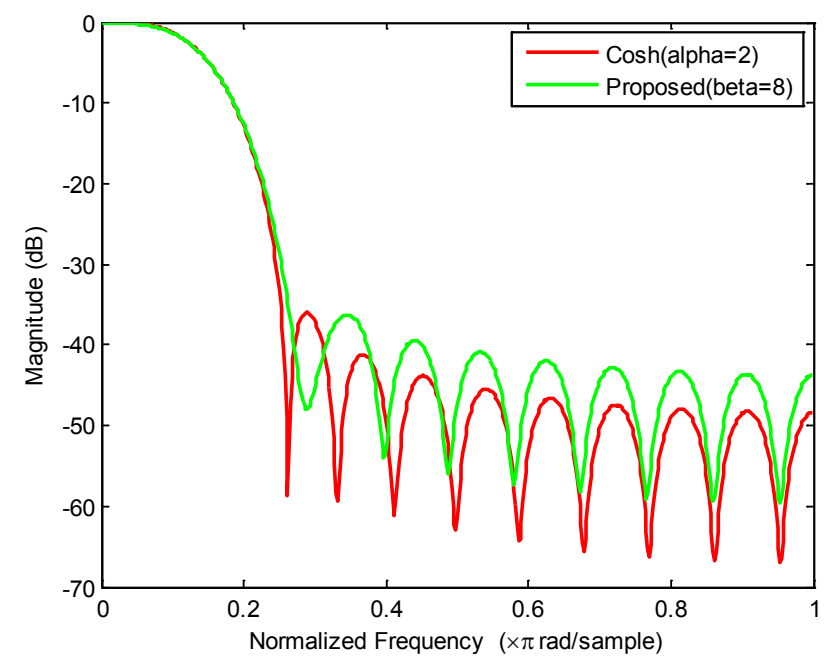

(c)

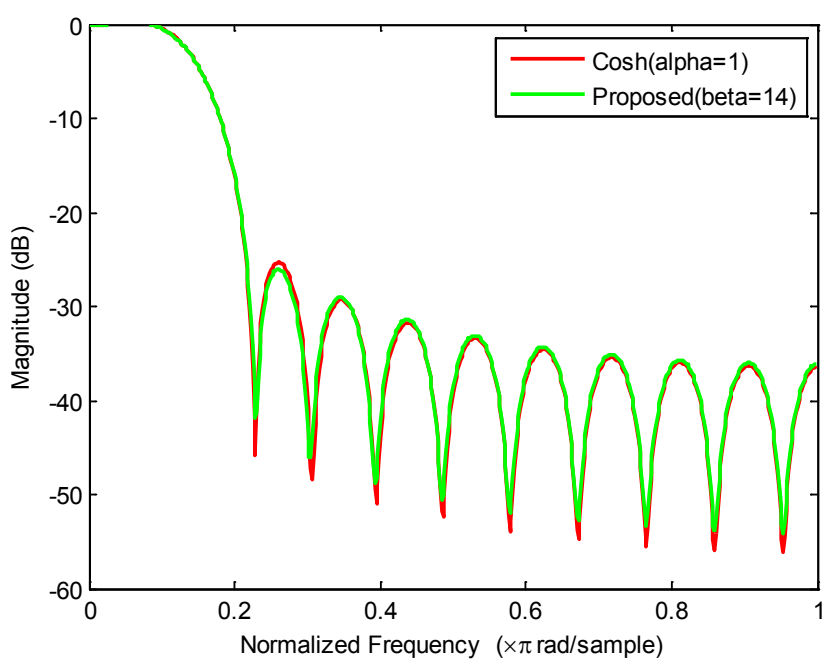

(b)

Figure 6. Characteristics comparison between Cosh window and proposed window for different values of $\alpha$ and $\beta$. (a) $\alpha=0$, $\beta=$ 50; (b) $\alpha=1, \beta=14$; (c) $\alpha=2, \beta=8$.

namely stopband attenuation and mainlobe width. From Figure 7(b) and Figure 7(c) better performance of our proposed window is easily visible.

Table 6 shows the resultant data obtained from the comparisons of exponential window and proposed window for $N=20$. From the tabulated data we observe that for $\alpha=0$ and $\beta=90$, proposed window provides both narrower main-lobe half width and greater minimum stopband attenuation compared to exponential window. For $\alpha=1$ and $\beta=13$, both of the windows have equal mainlobe half width but our proposed window provides increased stopband attenuation. For $\alpha=2$ and $\beta=10$, the resultant stopband attenuation of proposed window is greatly increased while the mainlobe half width is also reduced. For simplicity we take a small value of window length $(N=20)$. But the result would be the same for larger values also.

From the data placed in Table 5 and Table 6, we conclude that for the same window 


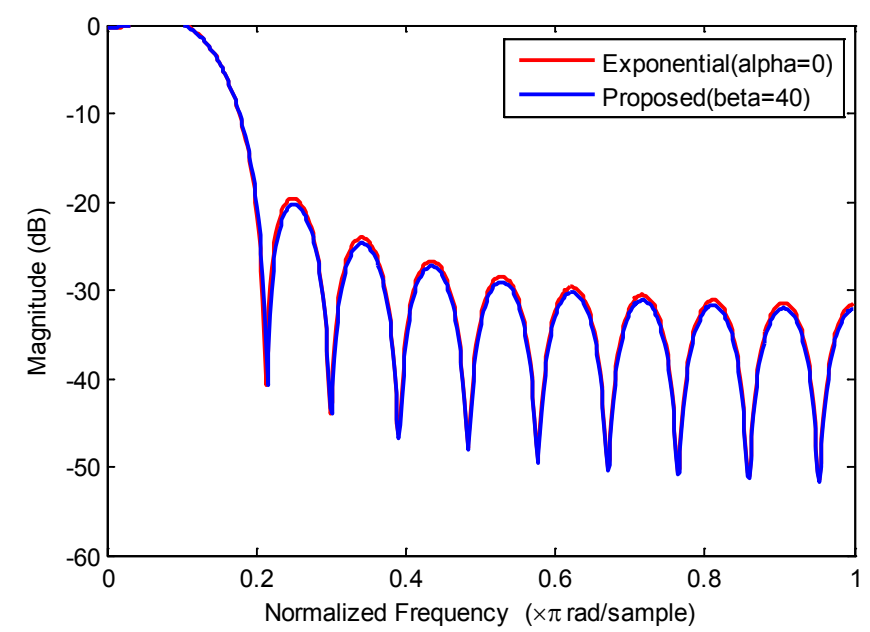

(a)

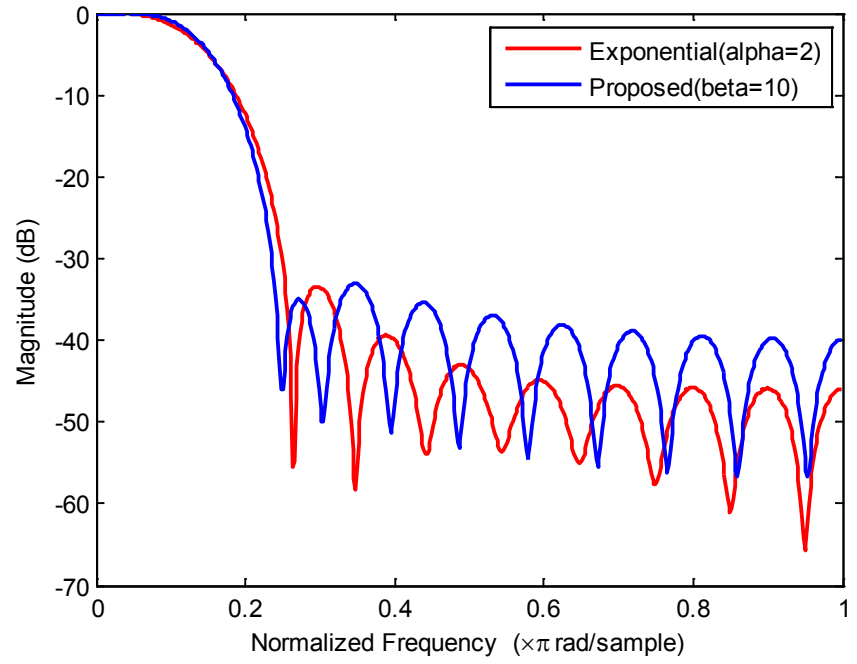

(c)

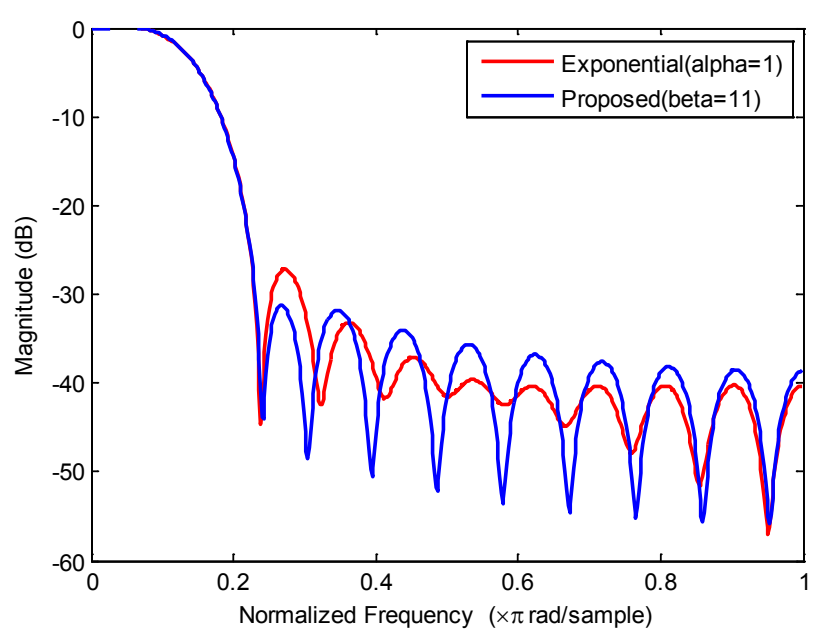

(b)

Figure 7. Characteristics comparison between exponential window and proposed window for different values of $\alpha$ and $\beta$. (a) $\alpha=$ $0, \beta=40$; (b) $\alpha=1, \beta=11$; (c) $\alpha=2, \beta=10$.

Table 6. Comparison data for exponential window and proposed window.

\begin{tabular}{cccc}
\hline Window & $N$ & $A s($ in dB $)$ & $W_{R}$ \\
\hline Exponential $(\alpha=0)$ & 20 & -24.65 & 0.154 \\
Proposed $(\beta=90)$ & 20 & -25.24 & 0.151 \\
Exponential $(\alpha=1)$ & 20 & -30.52 & 0.174 \\
Proposed $(\beta=13)$ & 20 & -32.14 & 0.174 \\
Exponential $(\alpha=2)$ & 20 & -36.50 & 0.200 \\
Proposed $(\beta=10)$ & 20 & -39.15 & 0.195 \\
\hline
\end{tabular}

length $N$, our proposed window remains superior in terms of both stopband attenuation and half mainlobe width. So our proposed can be a potential replacement for Cosh window and Exponential window for many important signal processing application 
where the length of the system cannot be increased (because this will increase the cost of the system) but better performance compared to the existing adjustable windows is required.

\section{Filtering Performance of Proposed Window}

In order to show the efficient performance of our proposed window, A MATLAB simulator is used to generate a signal $10 \cos (2 \pi n)$ mixed with high frequency component $2 \cos (20 \pi n+\pi / 4)$ shown in Figure 8(a). Clearly, this signal has a frequency component at $1 \mathrm{~Hz}$ and a frequency component at $10 \mathrm{~Hz}$. If we want to eliminate the high frequency component and the noise associated with it without affecting the low frequency component, we must have to use a lowpass filter having a cutoff frequency above $1 \mathrm{~Hz}$. Thus the mixed signal defined previously is applied to lowpass filter designed by using our proposed window. A cutoff frequency of $1.5 \mathrm{~Hz}$ and a filter length of $N=10$ has been selected. Figure 8(b) shows that the high frequency component and the associated noise above the cutoff frequency has been smoothly suppressed by our proposed window filter. Moreover, the amplitude of the low frequency component in the filtered signal remains unchanged.

\section{Conclusion}

In this paper we have proposed an adjustable window function and its performance characteristics are evaluated using MATLAB simulator. For most of the windowed filters, obtaining better minimum stopband attenuation is not possible without affecting the value of mainlobe half width. This contradictory behavior somehow limits the potential application of windowed filters. However our proposed window shows significant improvements in this regard. Within a particular range, this proposed window

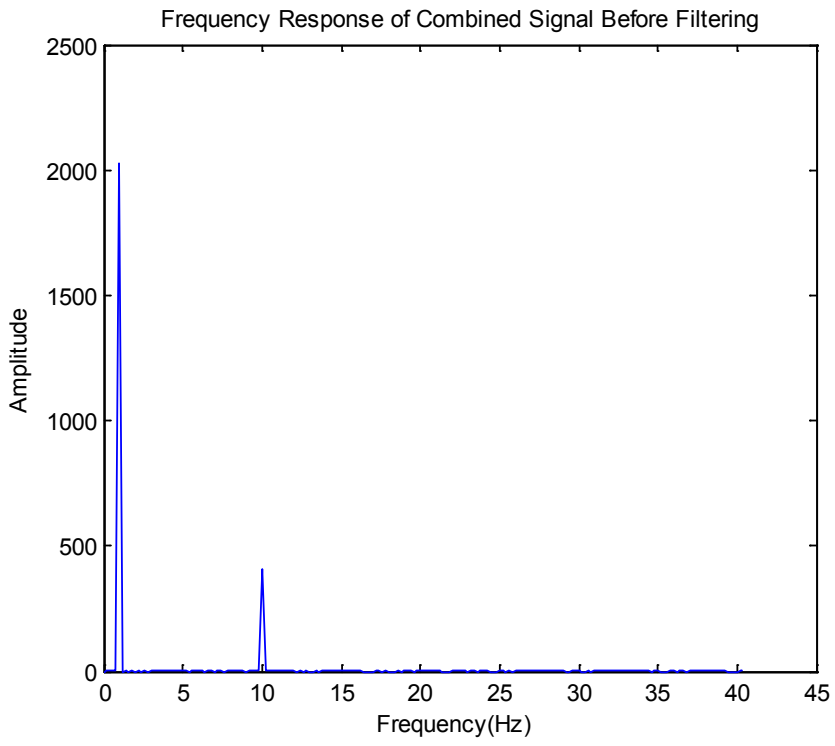

(a)

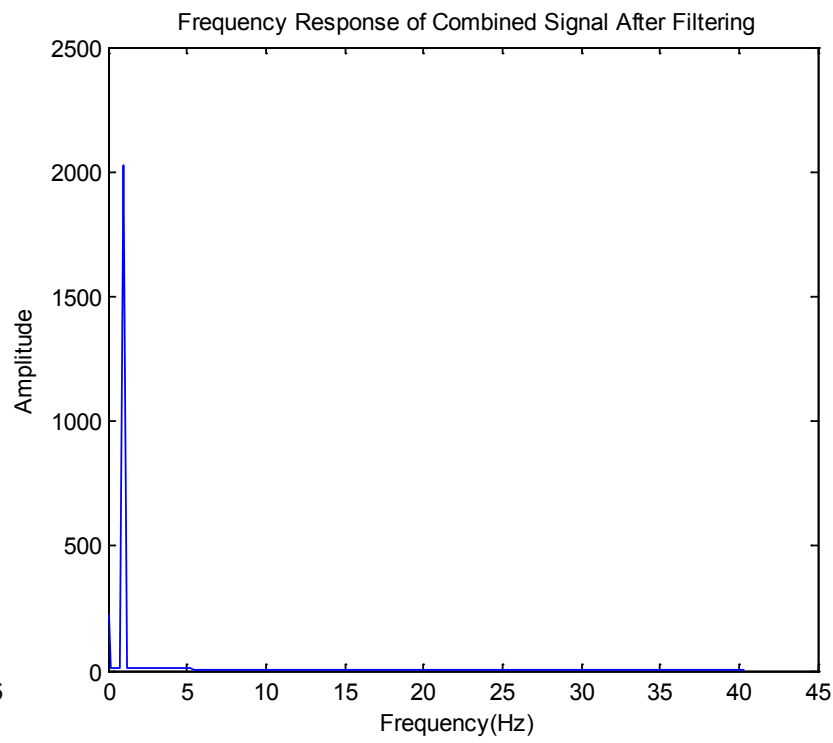

(b)

Figure 8. Filtration of high frequency signal by proposed window in the frequency domain: (a) before filtering; (b) after filtering. 
provides better stopband attenuation without compromising the value of mainlobe width. This improved performance makes it suitable for practical applications such as medical imaging systems.

\section{References}

[1] Smith, S.W. (1999) The Scientist and Engineer's Guide to Digital Signal Processing. 2nd Edition, California Technical Publication, 261-344.

[2] Bergen, S.W.A. and Antoniou, A. (2004) Design of Ultraspherical Window Functions with Prescribed Spectral Characteristics. EURASIP Journal of Applied Signal Processing, 13, 2053-2065. http://dx.doi.org/10.1155/S1110865704403114

[3] Rajput, S.S. and Bhadauria, S.S. (2012) Implementation of FIR Filter Using Adjustable Window Function and Its Application in Speech Signal Processing. International Journal of Advances in Electrical and Electronics Engineering, 1, 158-164.

[4] Rajput, S.S. and Bhadauria, S.S. (2012) Comparison of Band-Stop FIR Filter Using Modified Hamming Window and Other Window Functions and Its Application in Filtering a Mutitone Signal. International Journal of Advanced Research in Computer Engineering \& Technology (IJARCET), 1, 325-328.

[5] Bergen, S.W.A. and Antoniou, A. (2005) Design of Nonrecursive Digital Filters Using the Ultraspherical Window Function. EURASIP Journal on Applied Signal Processing, 12, 1910-1922. http://dx.doi.org/10.1155/ASP.2005.1910

[6] Samad, M.A. (2012) A Novel Window Function Yielding Suppressed Mainlobe Width and Minimum Sidelobe Peak. International Journal of Computer Science, Engineering and Information Technology (IJCSEIT), 2, 91-103. http://dx.doi.org/10.5121/ijcseit.2012.2209

[7] Avci, K. and Nacaroglu, A. (2008) A New Window Based on Exponential Function. Proceedings of the International Conference on Information \& Communication Technologies: from Theory to Applications, Damascus, Syria, 7-11 April 2008, 289-290. http://dx.doi.org/10.1109/rme.2008.4595727

[8] Nouri, M., Ghaemmaghami, S.S. and Falahati, A. (2011) An Improved Window Based on Cosine Hyperbolic Function. Journal of Selected Areas in Telecommunications (JSAT), July Edition, 8-11.

[9] Basokur, A.T. (2011) Designing Frequency Selective Filters via the Use of Hyperbolic Tangent Functions. Bulletin of the Earth Sciences Application and Research Centre of Hacettepe University, 32, 69-88.

[10] Basokur, A.T. (1998) Digital Filter Design Using the Hyperbolic Tangent Functions. Journal of the Balkan Geophysical Society, 1, 14-18. 